\title{
Geometric Quantum Computation
}

\author{
Artur Ekert \\ Marie Ericsson* \\ Patrick Hayden \\ Hitoshi Inamori \\ Jonathan A. Jones \\ Daniel K. L. Oi \\ Vlatko Vedral \\ Centre for Quantum Computation \\ University of Oxford \\ Clarendon Laboratory, Parks Road \\ Oxford OX1 3PU, UK
}

February 1, 2008

\begin{abstract}
We describe in detail a general strategy for implementing a conditional geometric phase between two spins. Combined with single-spin operations, this simple operation is a universal gate for quantum computation, in that any unitary transformation can be implemented with arbitrary precision using only single-spin operations and conditional phase shifts. Thus quantum geometrical phases can form the basis of any quantum computation. Moreover, as the induced conditional phase depends only on the geometry of the paths executed by the spins it is resilient to certain types of errors and offers the potential of a naturally fault-tolerant way of performing quantum computation.
\end{abstract}

\section{Introduction}

Among the surprising effects recently discovered in quantum mechanics is that a quantum system retains a memory of its motion when it undergoes a cyclic evolution [1]. This is reflected in the existence of the Berry phase, a phase acquired by the quantum state of the system in addition to the better known dynamic phase. The Berry phase is a purely geometrical effect that can be linked to the notion of parallel transport [2]: it depends only on the area covered by the motion of the system, and is independent of details of how the motion is executed. Berry phases have been demonstrated in a wide variety of systems [3], including NMR [4,5], the closely related technique of NQR [6 8], optical systems [9], and others.

*Department of Quantum Chemistry, Uppsala University, Box 518, Se-751 20 Uppsala, Sweden 
An equally exciting recent development in the field of quantum mechanics has been the discovery that quantum systems can be used to perform novel information processing tasks, including computations which are more efficient than any algorithm known on a classical computer [10 12]. Quantum information processing requires the ability to execute conditional dynamics 13 between two quantum bits (qubits), where the state of one qubit influences the evolution of another qubit during a quantum computation. Simple quantum information processing has been demonstrated using NMR [14 17] and trapped ions [18].

Recent experimental work has managed to combine these two quantum phenomena in the form of geometric quantum computation [19]. (For a more abstract approach see [20,21].) In this paper we seek to detail the theoretical ideas behind geometric quantum computation. In particular we show that Berry's phase may be used to implement conditional phase shifts, and thus any quantum gate [24]. We begin with brief introductions to both quantum gates and networks as well as to geometric phases, proceeding to analyse the dynamics of a spin-half system in order to see in detail how the theory of geometric phases applies there. Finally, we extend the ideas to pairs of spin-half particles, showing how to introduce a conditional geometric phase between the two particles.

\section{Phase gates and quantum computation}

\subsection{Qubits and networks}

A qubit is a quantum system in which the Boolean states 0 and 1 are represented by a prescribed pair of normalised and mutually orthogonal quantum states labeled as $\{|0\rangle,|1\rangle\}$. Unlike a simple Boolean variable, a qubit, typically a microscopic system such as an atom, a nuclear spin, or a polarised photon, can exist in an arbitrary superposition $\alpha|0\rangle+\beta|1\rangle$, making it more powerful as a computational resource.

In quantum computation, we set some register of qubits to an "input" state, evolve the qubits unitarily using simple building-block operations and then take the final state as "output". More formally, a quantum logic gate is a device which performs a fixed unitary operation on selected qubits in a fixed period of time and a quantum network is a device consisting of quantum logic gates whose computational steps are synchronised in time [23]. The outputs of some of the gates are connected by wires to the inputs of others. The size of the network is the number of gates it contains.

\subsection{Quantum logic gates}

The most common quantum gate is the Hadamard gate, a single qubit gate $H$ performing the unitary transformation known as the Hadamard transform. It is defined as

$$
H=\frac{1}{\sqrt{2}}\left(\begin{array}{cc}
1 & 1 \\
1 & -1
\end{array}\right) \quad|x\rangle-H-\frac{(-1)^{x}|x\rangle+|1-x\rangle}{\sqrt{2}}
$$


The matrix is written in the computational basis $\{|0\rangle,|1\rangle\}$ and the diagram on the right provides a schematic representation of the gate $H$ acting on a qubit in state $|x\rangle$, with $x=0,1$.

The addition of another single qubit gate, the phase shift gate $\phi$, defined as $|0\rangle \mapsto|0\rangle$ and $|1\rangle \mapsto e^{i \phi}|1\rangle$, or, in matrix notation,

$$
\phi=\left(\begin{array}{cc}
1 & 0 \\
0 & e^{i \phi}
\end{array}\right) \quad|x\rangle \longrightarrow e^{i x \phi}|x\rangle
$$

is actually sufficient to construct the following network (of size four), which generates the most general pure state of a single qubit (up to a global phase),

$$
|0\rangle \longrightarrow H \stackrel{2 \theta}{\longrightarrow} \overbrace{}^{\frac{\pi}{2}+\phi} \cos \theta|0\rangle+e^{i \phi} \sin \theta|1\rangle .
$$

Consequently, the Hadamard and phase gates are sufficient to construct any unitary operation on a single qubit.

Thus the Hadamard gates and the phase gates can be used to transform the input state $|0\rangle|0\rangle_{\ldots}|0\rangle$ of $n$ qubits into any state of the type $\left|\Psi_{1}\right\rangle\left|\Psi_{2}\right\rangle \ldots\left|\Psi_{n}\right\rangle$, where $\left|\Psi_{i}\right\rangle$ is an arbitrary superposition of $|0\rangle$ and $|1\rangle$. These are rather special $n$-qubit states, called the product states or the separable states. In general, a register of $n$ qubits can be prepared in states which are not separable, known as entangled states.

However, in order to entangle two or more qubits it is necessary to have access to two-qubit gates. One such gate is the controlled phase shift gate $B(\phi)$ defined as

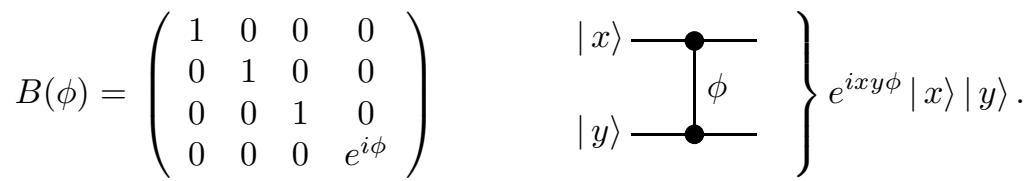

The matrix is written in the computational basis $\{|00\rangle,|01\rangle,|10\rangle,|11\rangle\}$ and the diagram on the right shows the structure of the gate.

\subsection{Universality}

An important result in the theory of quantum computation states that the Hadamard gate, and all $B(\phi)$ controlled phase gates form a universal set of gates: if the Hadamard gate as well as all $B(\phi)$ gates are available then any $n$-qubit unitary operation can be simulated exactly with less than $C 4^{n} n$ such gates, for some constant $C$ 24]. Consequently, being able to implement 1 and 2 -qubit phase gates is of crucial importance in quantum computation. In this paper we describe a new method for implementing the controlled phase gates based explicitly on geometric phases [1] 3] rather than dynamic ones. 


\section{Geometric phase}

\subsection{Cyclic evolution}

The states of a quantum system are usually described as being represented by vectors of norm $1\left(|\langle\psi \mid \psi\rangle|^{2}=1\right)$ in a complex Hilbert space $\mathcal{H}$. However, there is redundancy in this description since the state $|\psi\rangle$ is physically indistinguishable from the state $e^{i \phi}|\psi\rangle$. It is therefore convenient to consider instead the projective space $\mathcal{P}$, in which vectors are grouped into equivalence classes under the relation $|\psi\rangle \sim r e^{i \phi}|\psi\rangle$ for any $r>0$ and real $\phi$, thereby eliminating the ambiguity. The associated projection map is

$$
\begin{aligned}
\Pi: \mathcal{H} & \rightarrow \mathcal{P} \\
|\psi\rangle & \mapsto[|\psi\rangle]=\left\{\left|\psi^{\prime}\right\rangle:\left|\psi^{\prime}\right\rangle=r e^{i \phi}|\psi\rangle\right\} .
\end{aligned}
$$

If a system undergoes a cyclic evolution, the ket representing the system state traces out a path, $\mathcal{C}:[0, \tau] \longrightarrow \mathcal{H}$, where $\Pi(\mathcal{C})$ is a closed curve in $\mathcal{P}$, as illustrated in Figure 1. In other words, the initial and final states should be on the same ray in $\mathcal{H}$, but may be related by a phase, $e^{i \phi}$. We will measure this phase with respect to a reference curve in $\mathcal{H}$ : for each point $|\psi(t)\rangle$ on $\mathcal{C}$, we can choose a smoothly varying representative $|\tilde{\psi}(t)\rangle$ from $\Pi(\psi(t))$ in such a way that $|\tilde{\psi}(0)\rangle=|\tilde{\psi}(\tau)\rangle$. We can then write

$$
|\psi(t)\rangle=e^{i f(t)}|\tilde{\psi}(t)\rangle
$$

so that the phase change of $|\psi(0)\rangle$ associated with the cyclic evolution is given by $\phi=f(\tau)-f(0)$.

\subsection{Dynamic and geometric phase}

The time evolution of a quantum system is governed by the Schödinger equation,

$$
i \hbar \frac{\mathrm{d}}{\mathrm{d} t}|\psi(t)\rangle=H(t)|\psi(t)\rangle
$$

where $H(t)$ is the Hamiltonian. Substituting Eq. (6) into the above, rearranging and multiplying by $\langle\psi(t)|$ gives the following [25],

$$
\frac{\mathrm{d} f(t)}{\mathrm{d} t}=-\frac{1}{\hbar}\langle\psi(t)|H| \psi(t)\rangle+i\left\langle\tilde{\psi}(t)\left|\frac{\mathrm{d}}{\mathrm{d} t}\right| \tilde{\psi}(t)\right\rangle,
$$

or, when integrated,

$$
\phi=-\frac{1}{\hbar} \int_{0}^{\tau}\langle\psi(t)|H| \psi(t)\rangle \mathrm{d} t+i \int_{0}^{\tau}\left\langle\tilde{\psi}(t)\left|\frac{\mathrm{d}}{\mathrm{d} t}\right| \tilde{\psi}(t)\right\rangle \mathrm{d} t .
$$

Thus, $\phi$ can be decomposed into a dynamical phase

$$
\delta=-\frac{1}{\hbar} \int_{0}^{\tau}\langle\psi(t)|H| \psi(t)\rangle \mathrm{d} t
$$




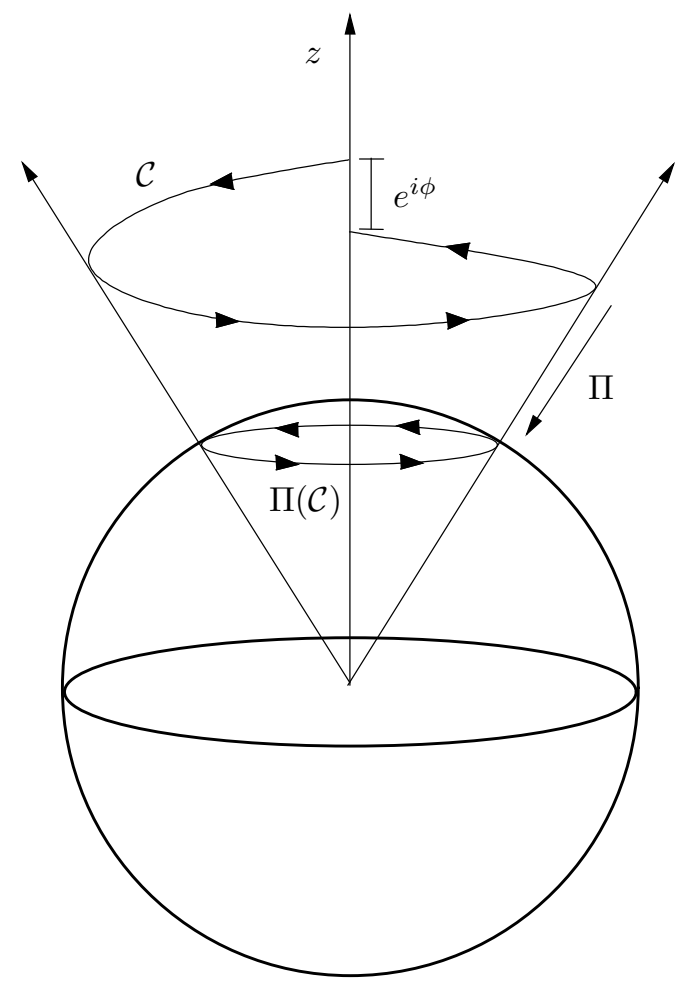

Figure 1: A schematic diagram of a spin-half particle undergoing a cyclic state evolution. Points on the sphere correspond to physically distinguishable states. Going through each point is a ray, on which phase information and normalisation is recorded. 
which depends on the Hamiltonian, and a geometric phase

$$
\gamma=i \oint_{\mathcal{C}}\langle\tilde{\psi}|\mathrm{d}| \tilde{\psi}\rangle
$$

which depends only on the path $\mathcal{C}$; $\gamma$ is independent of the rate at which $|\psi(t)\rangle$ progresses along $\mathcal{C}$, the Hamiltonian, or the choice of reference $\{|\tilde{\psi}\rangle\}$.

\subsection{Berry's phase}

A particular instance of this geometric phase is Berry's phase [1], which occurs when the adiabatic theorem (see [26]) is satisfied. In this case, if the initial state $|\psi(0)\rangle$ of the system is an eigenstate of the Hamiltonian, the state $|\psi(t)\rangle$ remains an eigenstate $|\psi(t)\rangle=|n(\mathbf{R})\rangle$ of the instantaneous Hamiltonian $H(\mathbf{R})$, where $\mathbf{R}$ is a set of time-varying parameters controlling the Hamiltonian. Supposing $\mathbf{R}$ traces a closed loop in the parameter space, the geometric phase of the system can be written in terms of $\mathbf{R}$. In this case, provided the energy eigenspace of the instantaneous Hamiltonians is non-degenerate along the path $\mathcal{C}$, the geometric phase acquired by the $n^{\text {th }}$-eigenstate is

$$
\gamma_{n}=i \oint_{C}\left\langle\widetilde{n(\mathbf{R})}\left|\nabla_{\mathbf{R}}\right| \widetilde{n(\mathbf{R})}\right\rangle \cdot d \mathbf{R}
$$

where $\nabla_{\mathbf{R}}$ is the gradient operator with respect to the parameters $\mathbf{R}$, and $|\widetilde{n(\mathbf{R})}\rangle$ is defined as in the previous section. This line integral can be transformed into a surface integral over any surface in the parameter space whose boundary is $\mathcal{C}$.

Since experimentally it is much easier to control the Hamiltonian than the actual state of a system, the adiabatic case is of importance. However, the adiabatic conditions necessarily mean that the processes take a long time compared to the characteristic dynamical time-scales, and thus are much slower than dynamic methods of generating phases.

\section{Single-qubit evolution}

\subsection{Qubit Dynamics}

Here we will focus on developing an understanding of the time evolution of a single qubit governed by a very general Hamiltonian. Recall that any $2 \times 2$ Hermitian matrix can be written in terms of the unit matrix and the three Pauli matrices. In particular a single qubit density operator can be parametrised as

$$
\rho=\frac{1}{2}(\mathbf{1}+\mathbf{s} \cdot \sigma)=\frac{1}{2}\left(\begin{array}{cc}
1+s_{z} & s_{x}-i s_{y} \\
s_{x}+i s_{y} & 1-s_{z}
\end{array}\right),
$$

where the real vector $\mathbf{s}=\left(s_{x}, s_{y}, s_{z}\right)$ is known as the Bloch vector. By the same token any $2 \times 2$ Hamiltonian can be written as

$$
H=\frac{\hbar}{2}\left(\Omega_{0} \mathbf{1}+\boldsymbol{\Omega} \cdot \sigma\right)
$$




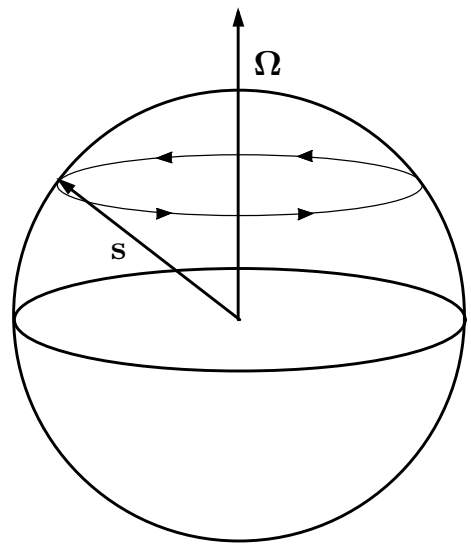

Figure 2: Solution to the equations of motion for a single spin-half particle.

where $\Omega$ is called the Rabi vector. Substituting Eq. (13) and Eq. (14) into the equation of motion for the density operator,

$$
i \hbar \frac{\mathrm{d}}{\mathrm{d} t} \rho=[H, \rho]
$$

and using the identity

$$
(\mathbf{a} \cdot \sigma)(\mathbf{b} \cdot \sigma)=(\mathbf{a} \cdot \mathbf{b}) \mathbf{1}+i(\mathbf{a} \times \mathbf{b}) \cdot \sigma,
$$

we find the following equation of motion for the Bloch vector,

$$
\frac{\mathrm{d}}{\mathrm{d} t} \mathbf{s}=\boldsymbol{\Omega} \times \mathbf{s} .
$$

This equation has a simple geometric solution: vector $\mathbf{s}$ revolves around vector $\boldsymbol{\Omega}$ with angular frequency given by $|\boldsymbol{\Omega}|$, the length of $\boldsymbol{\Omega}$, as illustrated in Figure 2.

From Eq. (17), it is relatively easy to move to situations typical of those encountered in quantum computation. Hamiltonians that describe qubits interacting with external potentials are usually time dependent. Typical external perturbations are periodic such as, for example, spins coupled to oscillating magnetic fields in NMR or atomic dipole moments coupled to oscillating electromagnetic field in the optical domain. Within the Rotating Wave Approximation the oscillating field can be replaced by a rotating field, and so the Hamiltonian is of the form

$$
H(t)=\frac{\hbar}{2}\left(\begin{array}{cc}
\omega_{0} & \omega_{1} e^{-i(\omega t+\phi)} \\
\omega_{1} e^{i(\omega t+\phi)} & -\omega_{0}
\end{array}\right),
$$

where $\omega_{0} / 2 \pi$ is the system's transition frequency, while $\omega / 2 \pi$ and $\hbar \omega_{1}$ are the frequency and the amplitude of the oscillating field, respectively. This gives

$$
\Omega_{x}=\omega_{1} \cos (\omega t+\phi), \quad \Omega_{y}=\omega_{1} \sin (\omega t+\phi), \quad \Omega_{z}=\omega_{0} .
$$


In order to solve Eq. (17) it is convenient to consider the evolution of $\mathbf{s}$ in a frame which rotates with frequency $\omega$ around the $z$-axis. More precisely, we write

$$
\mathbf{s}(t)=R_{z}(\omega t) \mathbf{s}^{\prime}(t), \quad \boldsymbol{\Omega}(t)=R_{z}(\omega t) \boldsymbol{\Omega}^{\prime}(t),
$$

where $R_{z}(\omega t)$ is the rotation matrix

$$
R_{z}(\omega t)=\left(\begin{array}{ccc}
\cos (\omega t) & -\sin (\omega t) & 0 \\
\sin (\omega t) & \cos (\omega t) & 0 \\
0 & 0 & 1
\end{array}\right)=\exp \left(\omega t M_{z}\right)
$$

for

$$
M_{z}=\left(\begin{array}{ccc}
0 & -1 & 0 \\
1 & 0 & 0 \\
0 & 0 & 0
\end{array}\right) .
$$

Substituting Eq. (20) into Eq. (17) and taking into account that

$$
\frac{\mathrm{d}}{\mathrm{d} t} R_{z}(\omega t)=R_{z}(\omega t)\left(\omega M_{z}\right), \quad M_{z} \mathbf{s}^{\prime}=\hat{\mathbf{z}} \times \mathbf{s}^{\prime},
$$

where $\hat{\mathbf{z}}$ is a unit vector in the $z$-direction, we obtain

$$
\frac{\mathrm{d}}{\mathrm{d} t} \mathbf{s}^{\prime}=\boldsymbol{\Omega}^{\prime} \times \mathbf{s}^{\prime}
$$

with the time-independent vector $\boldsymbol{\Omega}^{\prime}$,

$$
\Omega_{x}^{\prime}=\omega_{1} \cos (\phi), \quad \Omega_{y}^{\prime}=\omega_{1} \sin (\phi), \quad \Omega_{z}^{\prime}=\omega_{0}-\omega .
$$

If we can control the strength of coupling $\hbar \omega_{1}$, the frequency $\omega$ and the phase $\phi$ of the external field we can prepare any vector $\boldsymbol{\Omega}$. This implies that if we know the initial state of the qubit then with a single rotation we can position the Bloch vector $\mathbf{s}$ in any prescribed direction.

\subsection{Calculating geometric phases}

We can now apply the results of the previous section to the task of developing a geometric phase of a spin-half particle located in an external oscillating field. By varying the parameters of the Hamiltonian adiabatically we will send a qubit through a cyclic evolution, whose associated geometric phase can be calculated using Eq. (11).

Working in the rotating frame, the components $\left(\Omega_{x}^{\prime}, \Omega_{y}^{\prime}, \Omega_{z}^{\prime}\right)$ of the Hamiltonian are given by Eq. (25), where the frequency and power of the rotating field can be used to control the value of the angle $\theta$ of Figure 3. In the absence of a rotating field the Rabi vector lies along the $z$-axis, and as the power of the rotating field is slowly increased from zero to $\omega_{1}$ the vector tilts towards the $x y$-plane. If the Bloch vector $\mathbf{s}^{\prime}$ is initially aligned with $\boldsymbol{\Omega}^{\prime}$ then by the adiabatic theorem, it will remain aligned with $\boldsymbol{\Omega}^{\prime}$ provided $\boldsymbol{\Omega}^{\prime}$ varies slowly. Therefore, 


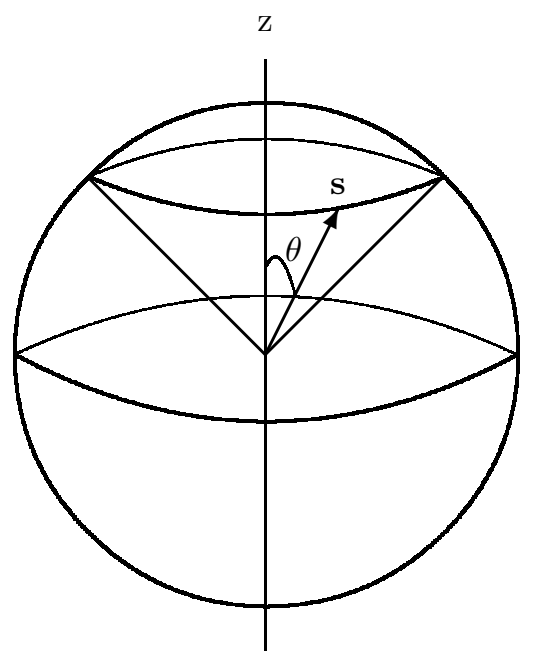

Figure 3: Spin-half particle in a magnetic field

from the relation between the different components $\boldsymbol{\Omega}^{\prime}$ we find that the angle $\theta$ between the Bloch vector and the $z$-axis will be

$$
\cos \theta=\frac{\Omega_{z}^{\prime}}{\sqrt{\Omega_{x}^{\prime 2}+\Omega_{y}^{\prime 2}+\Omega_{z}^{\prime 2}}}=\frac{\omega_{0}-\omega}{\sqrt{\left(\omega_{0}-\omega\right)^{2}+\omega_{1}^{2}}} .
$$

Varying the phase $\phi$ of the rotating field from Eq. (25) will then cause $\mathbf{s}^{\prime}$ to rotate around the $z$-axis. The geometric phase associated with a full revolution is easily calculated by parameterizing the state in terms of the $|\uparrow\rangle$ and $|\downarrow\rangle$ eigenstates of quantization along the $z$-axis, $|\tilde{\psi}(\alpha)\rangle=\cos (\theta / 2)|\uparrow\rangle+$ $\sin (\theta / 2) e^{i \alpha}|\downarrow\rangle$, where $\alpha$ changes smoothly from 0 to $2 \pi$. Using Eq. (11),

$$
\begin{aligned}
\gamma & =i \oint_{\mathcal{C}}\left(\cos \frac{\theta}{2}|\uparrow\rangle+\sin \frac{\theta}{2} e^{i \alpha}|\downarrow\rangle\right)^{\dagger} \mathrm{d}\left(\cos \frac{\theta}{2}|\uparrow\rangle+\sin \frac{\theta}{2} e^{i \alpha}|\downarrow\rangle\right) \\
& =-\int_{0}^{2 \pi}\left(\cos \frac{\theta}{2}\langle\uparrow|+\sin \frac{\theta}{2} e^{-i \alpha}\langle\downarrow|\right)\left(\sin \frac{\theta}{2} e^{i \alpha}|\downarrow\rangle\right) \mathrm{d} \alpha \\
& =-\frac{1}{2}(1-\cos \theta) \int_{0}^{2 \pi} \mathrm{d} \alpha \\
& =-\pi(1-\cos \theta) .
\end{aligned}
$$

Furthermore, the result can be generalized to any closed path with the result that the geometric phase is equal to half the solid angle enclosed by $\mathcal{C}$ on the Bloch sphere [1]. 


\subsection{Eliminating dynamic phases}

In order to perform conditional quantum gate operations using geometric phases only, it is necessary to find a way to eliminate the dynamic phase. One approach is to use a refocussing technique known as spin-echo. The basic idea is to apply the cyclic evolution twice, with the second application surrounded by a pair of fast $\pi$ transformations (this being simply the transformation that swaps the basis states $|\uparrow\rangle$ and $|\downarrow\rangle$.) The net effect of this compound transformation would be to cancel all the acquired phases except that the second cyclic evolution is performed by retracing the first but in the opposite direction so that while the dynamic phases cancel, the geometric phases do not.

To see why this is so, let $\mathcal{C}_{\uparrow}$ be the closed curve in $\mathcal{P}$ traced out by $|\tilde{\uparrow}\rangle$ during the first cyclic evolution and $\mathcal{C}_{\downarrow}$ the one traced out by $|\tilde{\downarrow}\rangle$, with corresponding dynamic and geometric phases $\delta_{\uparrow}, \gamma_{\uparrow}, \delta_{\downarrow}$, and $\gamma_{\downarrow}$. Referring back to Eq. (27) we see that $\gamma_{\uparrow}=-\gamma$ and $\gamma_{\downarrow}=\gamma$ for $\gamma=\pi(1-\cos \theta)$. Similarly, if we write $\overline{\mathcal{C}}_{\uparrow}$ and $\overline{\mathcal{C}}_{\downarrow}$ for the second cyclic evolution, these are simply $\mathcal{C}_{\uparrow}$ and $\mathcal{C}_{\downarrow}$ carried out in opposite orientations so they have corresponding phases $\bar{\gamma}_{\uparrow}=\gamma$ and $\bar{\gamma}_{\downarrow}=-\gamma$.

In summary, we can follow the states through the compound evolution as follows:

$$
\begin{aligned}
& |\uparrow\rangle \stackrel{\mathcal{C}_{\uparrow}}{\longrightarrow} e^{i\left(\delta_{\uparrow}-\gamma\right)}|\uparrow\rangle \stackrel{\pi}{\longrightarrow} e^{i\left(\delta_{\uparrow}-\gamma\right)}|\downarrow\rangle \stackrel{\overline{\mathcal{C}}_{\downarrow}}{\longrightarrow} e^{i\left(\delta_{\uparrow}+\delta_{\downarrow}-2 \gamma\right)}|\downarrow\rangle \stackrel{\pi}{\longrightarrow} e^{i\left(\delta_{\uparrow}+\delta_{\downarrow}-2 \gamma\right)}|\uparrow\rangle \\
& |\downarrow\rangle \stackrel{\mathcal{C}_{\downarrow}}{\longrightarrow} e^{i\left(\delta_{\downarrow}+\gamma\right)}|\downarrow\rangle \stackrel{\pi}{\longrightarrow} e^{i\left(\delta_{\downarrow}+\gamma\right)}|\uparrow\rangle \stackrel{\overline{\mathcal{C}}_{\uparrow}}{\longrightarrow} e^{i\left(\delta_{\uparrow}+\delta_{\downarrow}+2 \gamma\right)}|\uparrow\rangle \stackrel{\pi}{\longrightarrow} e^{i\left(\delta_{\uparrow}+\delta_{\downarrow}+2 \gamma\right)}|\downarrow\rangle .
\end{aligned}
$$

Since the global phase factor $e^{i\left(\delta_{\uparrow}+\delta_{\downarrow}\right)}$ is not physical this sequence of operations behaves as promised. The dynamic phases are eliminated and we are left with an exclusively geometric phase difference of $4 \gamma=4 \pi \cos \theta$.

\section{Conditional dynamics}

\subsection{2-Spin Hamiltonian}

This geometric phase can be used to implement a 2-qubit controlled-phase gate. Consider to begin with a system of two non-interacting spin-half particles $S_{a}$ and $S_{b}$. In a reference frame aligned with the static field, the Hamiltonian reads

$$
H_{0}=\hbar \omega_{a} S_{a z} \otimes \mathbf{1}_{b}+\hbar \omega_{b} \mathbf{1}_{a} \otimes S_{b z},
$$

or, in the basis $\left\{\left|S_{a z}, S_{b z}\right\rangle\right\}_{S_{a z}, S_{b z}}=\{|\uparrow \uparrow\rangle,|\uparrow \downarrow\rangle,|\downarrow \uparrow\rangle,|\downarrow \downarrow\rangle\}$,

$$
H_{0}=\frac{\hbar}{2}\left(\begin{array}{cccc}
\omega_{a}+\omega_{b} & 0 & 0 & 0 \\
0 & \omega_{a}-\omega_{b} & 0 & 0 \\
0 & 0 & -\omega_{a}+\omega_{b} & 0 \\
0 & 0 & 0 & -\omega_{a}-\omega_{b}
\end{array}\right)
$$

where the frequencies $\omega_{a} / 2 \pi$ and $\omega_{b} / 2 \pi$ are the transition frequencies of the two spins and we have used the scaled Pauli operators $S_{i}=\sigma_{i} / 2$. (From now on we assume that $\omega_{a}$ and $\omega_{b}$ are very different with $\omega_{a}>\omega_{b}$.) 


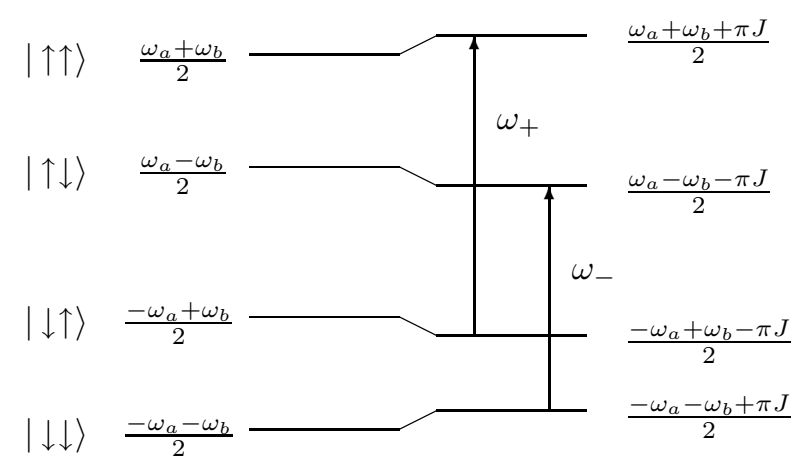

Figure 4: The energy diagram of two interacting spin-half nuclei. The transition frequency of the first spin depends on the state of the second spin.

If the two particles are sufficiently close to each other, they will interact, creating additional splittings between the energy levels. In the case of two spinhalf particles, the magnetic field of one spin may directly or indirectly affect the energy levels of the other spin; the energy of the system is increased by $\pi \hbar J / 2$ if the spins are parallel and decreased by $\pi \hbar J / 2$ if the spins are antiparallel. The Hamiltonian of the system taking into account this interaction reads

$$
H=H_{0}+2 \pi \hbar J S_{a z} \otimes S_{b z},
$$

or, in the previously chosen basis,

$$
H=\frac{\hbar}{2}\left(\begin{array}{cccc}
\omega_{a}+\omega_{b}+\pi J & 0 & 0 & 0 \\
0 & \omega_{a}-\omega_{b}-\pi J & 0 & 0 \\
0 & 0 & -\omega_{a}+\omega_{b}-\pi J & 0 \\
0 & 0 & 0 & -\omega_{a}-\omega_{b}+\pi J
\end{array}\right) .
$$

Figure 1 illustrates the energy levels of the system. When spin $S_{b}$ is in state $|\uparrow\rangle$, the transition frequency of the spin $S_{a}$ is

$$
\omega_{+}=\omega_{a}+\pi J,
$$

whereas when spin $S_{b}$ is in state $|\downarrow\rangle$, the transition frequency of the spin $S_{a}$ is

$$
\omega_{-}=\omega_{a}-\pi J
$$

\subsection{Conditional phase shift}

Now suppose that in addition to the static field, we apply a rotating field that is slowly varied as described in Section 4.2. We have seen that the Berry phase 
acquired by a spin depends on its transition resonance frequency as given by Eq. (26). Therefore, at the end of a cyclic evolution, the Berry phase acquired by the spin $S_{a}$ will be different for the two possible states of spin $S_{b}$. Indeed, when spin $S_{b}$ is in state $|\uparrow\rangle$, the Berry phase acquired by the spin $S_{a}$ is $\gamma_{+}=$ $\mp \pi\left(1-\cos \theta_{+}\right)$, with the sign negative or positive depending on whether spin $S_{a}$ is up or down, respectively, and

$$
\cos \theta_{+}=\frac{\omega_{+}-\omega}{\sqrt{\left(\omega_{+}-\omega\right)^{2}+\omega_{1}^{2}}} .
$$

Similarly, when spin $S_{b}$ is in state $|\downarrow\rangle$, the Berry phase acquired by the spin $S_{a}$ is $\gamma_{-}=\mp \pi\left(1-\cos \theta_{-}\right)$where

$$
\cos \theta_{-}=\frac{\omega_{-}-\omega}{\sqrt{\left(\omega_{-}-\omega\right)^{2}+\omega_{1}^{2}}} .
$$

As in the single-particle case, it is necessary to eliminate the dynamic phase in order to construct a purely geometric conditional phase gate. This can be accomplished using almost the same technique as in the single-particle case described in Section 4.3. In this case, however, we must apply the sequence of operations

$$
\mathcal{C} \longrightarrow \pi_{a} \longrightarrow \overline{\mathcal{C}} \longrightarrow \pi_{b} \longrightarrow \mathcal{C} \longrightarrow \pi_{a} \longrightarrow \overline{\mathcal{C}} \longrightarrow \pi_{b}
$$

where $\pi_{a}$ and $\pi_{b}$ are $\pi$-pulses applied to particles $a$ and $b$, respectively, and $\mathcal{C}$ and

$\overline{\mathcal{C}}$ are adiabatic transformations as in Section 4.3. If we define the differential Berry phase shift

$$
\Delta \gamma=\gamma_{+}-\gamma_{-}=\pi\left(\frac{\omega_{+}-\omega}{\sqrt{\left(\omega_{+}-\omega\right)^{2}+\omega_{1}^{2}}}-\frac{\omega_{-}-\omega}{\sqrt{\left(\omega_{-}-\omega\right)^{2}+\omega_{1}^{2}}}\right)
$$

then the net transformation, up to global phases, is given by

$$
\left(\begin{array}{cccc}
e^{2 i \Delta \gamma} & 0 & 0 & 0 \\
0 & e^{-2 i \Delta \gamma} & 0 & 0 \\
0 & 0 & e^{-2 i \Delta \gamma} & 0 \\
0 & 0 & 0 & e^{2 i \Delta \gamma}
\end{array}\right) .
$$

Thus, we have succeeded in engineering a conditional evolution since the state of the qubit $S_{b}$ influences the phase acquired by a second qubit $S_{a}$. 27] This gate, which introduces a phase of $e^{2 i \Delta \gamma}$ if the two spins are aligned and $e^{-2 i \Delta \gamma}$ if they are anti-aligned, is equivalent to the controlled phase gate introduced in Section 2 [22].

\subsection{Fault Tolerance}

The form of the dependence of $\Delta \gamma$ on the detuning $\omega_{a}-\omega$ and the amplitude of the oscillating field $\omega_{1}$ builds into the geometric phase gate a natural type of 


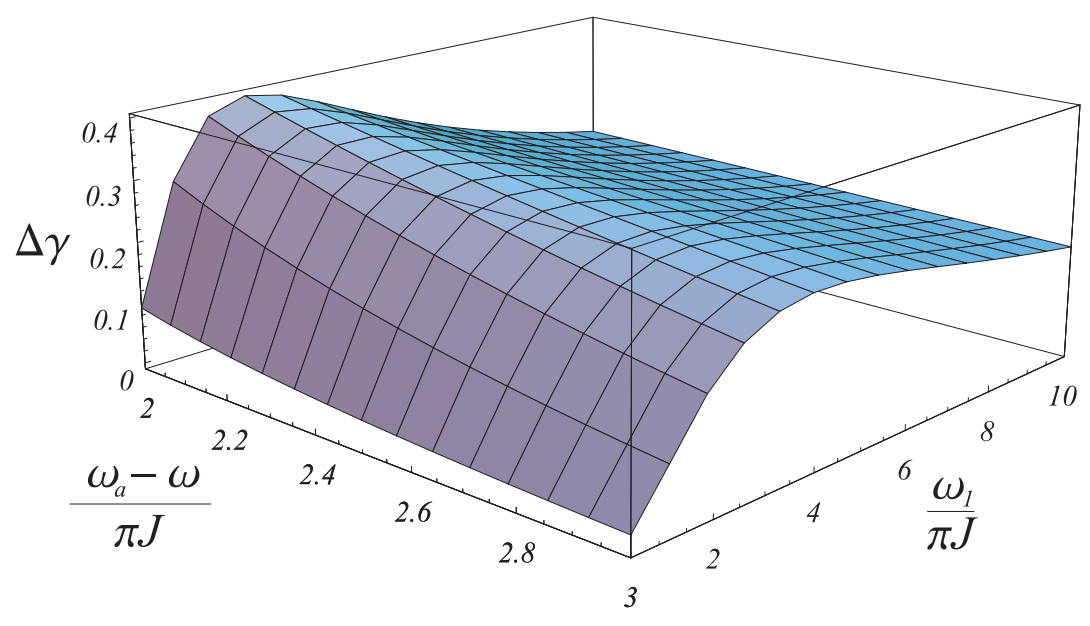

Figure 5: Plot of differential phase shift $\Delta \gamma$ as a function of $\frac{\omega_{a}-\omega}{\pi J}$ and $\frac{\omega_{1}}{\pi J}$.

fault tolerance not present in the simple non-geometric conditional phase gate. In many experiments, such as NMR, it is easy to control the detuning quite precisely, but relatively difficult to control the amplitude of the oscillating field. Figure 5 plots $\Delta \gamma$ as a function of these parameters in units of $\pi J$ for a range of values: for fixed $\omega_{a}-\omega$, we see that there is a peak in $\Delta \gamma$ as a function of $\omega_{1}$. Therefore, if $\omega_{1}$ is chosen to coincide with this peak then $\Delta \gamma$ will be insensitive to errors in $\omega_{1}$ to first order. As the height of the peak depends on the detuning, any desired controlled phase gate can be achieved.

\section{Conclusions}

The techniques described in this paper constitute a novel approach to quantum computation, one that builds entangling gates entirely out of conditional geometric phases. These techniques are readily implementable with current technology in quantum optics and have already been demonstrated by some of the authors using NMR [19]. It would be interesting to further analyse the robustness of geometric quantum computation to errors. While it has been observed that geometric phases are robust to certain types of noise in the classical parameters controlling the Hamiltonian, it has not been determined how geometric phases behave in the presence of decoherence or depolarisation of the quantum system. 


\section{Acknowledgments}

This work was supported in part by the European TMR Research Network ERP-4061PL95-1412, The Royal Society of London, Elsag, Starlab (Riverland NV, Belgium), the European Science Foundation, CESG, and the Rhodes Trust.

\section{References}

[1] M.V. Berry, 1984, Proc. Roy. Soc. A 392, pp.47.

[2] B. Simon, 1983, Phys. Rev. Lett. 51, pp.2167.

[3] A. Shapere and F. Wilczek, 1989, Geometric phases in Physics, World Scientific, Singapore.

[4] D. Suter, G. Chingas, R. Harris and A. Pines, 1987, Molec. Phys. 61, pp.1327.

[5] M. Goldman, V. Fleury and M. Guéron, 1996, J. Magn. Reson. A 118, pp.11.

[6] R. Tycko, 1987, Phys. Rev. Lett. 58, pp.2281.

[7] S. Appelt, G. Wäckerle and M. Mehring, 1994 Phys. Rev. Lett. 72, pp.3921.

[8] J. A. Jones and A. Pines, 1997, J. Chem. Phys. 106, pp.3007.

[9] A. Tomita and R. Chiao, 1986, Phys. Rev. Lett. 57, pp.937.

[10] D. Deutsch, 1985, Proc. R. Soc. Lond. A 400, pp.97.

[11] P. W. Shor, 1994, Proc. 35th Ann. Symp. on Fund. of Comp. Sci..

[12] L. Grover, 1996, Proc. 28th Ann. Symp. on the Th. of Comp. pp.212.

[13] A. Barenco, D. Deutsch, A. Ekert and R. Jozsa, 1995, Phys. Rev. Lett. 74, pp.4083.

[14] D. G. Cory, A. F. Fahmy, and T. F. Havel, 1996, in "PhysComp '96" (T. Toffoli, M. Biafore, and J. Leão, Eds.), New England Complex Systems Institute pp.87-91.

[15] D. G. Cory, A. F. Fahmy and T. F. Havel, 1997, Proc. Nat. Acad. Sci. USA 94, pp.1634.

[16] N. A. Gershenfeld and I. L. Chuang, 1997, Science 275, pp.350.

[17] J. A. Jones and M. Mosca, 1998, J. Chem. Phys. 109, pp.1648.

[18] C. Monroe, D. M. Meekhof, B. E. King, W. M. Itano and D. J. Wineland, 1995, Phys. Rev. Lett. 75, pp.4714. 
[19] J. A. Jones, V. Vedral, A. Ekert, and G. Castagnoli, 2000, Nature 403 pp.869.

[20] P. Zanardi, M. Rasetti, 1999, Phys. Lett. A 264 pp.94.

[21] J. Pachos, P. Zanardi, and M. Rasetti, Phys. Rev. A In press.

[22] J. A. Jones, R. H. Hansen and M. Mosca, 1998, J. Magn. Reson. 135, pp.353.

[23] D. Deutsch, 1989, Proc. R. Soc. Lond. A 425, pp.73.

[24] A. Barenco, C. H. Bennett, R. Cleve, D. P. DiVincenzo, N. Margolus, P. W. Shor, T. Sleator, J. Smolin and H.Weinfurter, 1995, Phys. Rev. A 52, pp.3457.

[25] J. Anandan, 1992, Nature 360 pp.307.

[26] A. Galindo and P. Pascual, 1990, Quantum Mechanics II Springer-Verlag.

[27] Animated representations of the single qubit and conditional gates can be found at http://www.qubit.org/research/Theory/Animations. 